\title{
KOSZUL RESOLUTIONS
}

BY

\author{
STEWART B. PRIDDY( $\left.{ }^{(}\right)$
}

\begin{abstract}
Resolutions, which generalize the classical Koszul resolutions, are constructed for a large class of augmented algebras including the Steenrod algebra and the universal enveloping algebras. For each such algebra $A$, an explicit differential algebra $\bar{K}^{*}(A)$ is described such that $(1) \bar{K}^{*}(A)$ is a small quotient algebra of the cobar complex and (2) the homology of $\bar{K}^{*}(A)$ is the cohomology algebra $H^{*}(A)$. The resolution of May for restricted Lie algebras in characteristic 2 is retrieved and a simple derivation of the resolution of Kan et al. of the Steenrod algebra is given.
\end{abstract}

The purpose of this paper is to construct resolutions for a large class of algebras which includes the Steenrod algebra and the universal enveloping algebras.

It is a basic problem of homological algebra to compute the cohomology algebras of various augmented algebras. Unfortunately, the canonical tool for attacking this problem - the bar resolution-is often intractable. In some instances, however, one is able to find a simpler resolution. For example, a classical result states that the cohomology algebra of a Lie algebra $L$ may be computed using the Koszul resolution $=U(L) \otimes E(L)$, with an appropriate differential, where $U(L)$ is the universal enveloping algebra of $L$ and $E(L)$ is the exterior algebra of $L$ [2, Chapter 8, §7]. This resolution is particularly nice because (1) it is a subcomplex of the bar resolution, and (2) it is much smaller than the bar resolution (i.e., has fewer generators). Our resolutions are conceptually analogous and so we call them Koszul resolutions and the algebras for which they are defined Koszul algebras. Our theory subsumes May's generalization of the classical Koszul resolution and his resolution for a restricted Lie algebra in characteristic 2 [4]. In the (motivating) case of the Steenrod algebra we also retrieve the resolution of Kan et al. [1].

The paper is divided into 10 sections, the first of which introduces notation and terminology. In $\S 2$ we define the notion of a Koszul algebra. The principal examples of the paper are given here along with an explicit determination of the cohomology algebras of homogeneous Koszul algebras. $\$ 3$ is devoted to constructing resolutions for Koszul algebras. Dual complexes are studied in $\S 4$. In particular, given a set of generators and relations for a Koszul algebra $A$ we give a set of generators and relations for a differential algebra whose homology algebra is $H^{*} A$. $\$ 5$ introduces

Received by the editors May 8, 1969.

AMS subject classification. Primary 1820; Secondary 5534.

Key words and phrases. Koszul resolutions, bar and cobar resolutions, cohomology of the Steenrod algebra, cohomology of Lie algebras.

(1) Partially supported by NSF GP-8217.

Copyright (C) 1970, American Mathematical Society 
the concept of a Poincaré-Birkhoff-Witt algebra and shows that any such algebra is a Koszul algebra. In $\$ \$ 6$ and 7 we retrieve the results of May and Kan et al. mentioned above. The role of matric Massey products in the theory is briefly examined in $\S 8$. For certain Koszul algebras $A$ there is a natural identification of $A$ and $H^{*} H^{*} A$. $\S 9$ studies this duality and its relation to a dual Adams spectral sequence. The last section is devoted to the proof of the main result of $\S 3$.

The principal results of this paper were announced in [9]. The author wishes to thank D. M. Kan for several conversations and to especially thank J. P. May whose suggestions have improved the definitional structure and organization of the paper. Also, the author thanks A. F. Lawrence and M. E. Mahowald for fruitful discussions which prompted the analysis of 7.1(1).

Finally, while the results of this paper are intended for application to the graded algebras arising from algebraic topology, it is also possible to obtain the classical Koszul resolutions used by the algebraists; e.g., the resolution of a cyclic group [2] and Tate's resolutions for noetherian and local rings [11]. We leave this for the interested reader.

1. Preliminaries. The purpose of this section is to give a reasonably complete account of the notions of homological algebra necessary for this paper-for more complete details the reader is referred to MacLane [3] and May [4].

All modules $M=\left\{M_{p}\right\}$ are $Z$-graded (or multigraded) of finite type over a field $F$. In addition all modules have a positive (or negative) gradation; i.e., $M_{p}=0$ for $p<0$ (or $M_{p}=0$ for $p>0$ ). If $M$ has a negative gradation we shall reindex $M$ using upper indices $M^{n}=M_{-n}$. The elements $m \in M_{p}$ are said to have degree $p(\operatorname{deg} m=p)$. In case $M$ is multigraded the degree is the total degree (e.g., if $m \in M_{p, q}$ then $\operatorname{deg} m=p+q$ ). We shall adhere to the standard sign convention: if objects $a$ and $b$ are permuted then the $\operatorname{sign}(-1)^{\operatorname{deg} a \cdot \operatorname{deg} b}$ is introduced.

Let $M^{*}$ denote the graded $F$-dual of $M$ given by $M^{* p}=\operatorname{Hom}\left(M_{p}, F\right)$. Recall the following identification isomorphisms:

(1.1) $\varphi: M \rightarrow M^{* *}$

given by $\varphi(m)(f)=(-1)^{\operatorname{deg} f \operatorname{deg} m} f(m)$ and

(1.2) $\theta: M^{*} \otimes N^{*} \rightarrow(M \otimes N)^{*}$

given by $\theta(f \otimes g)(m \otimes n)=(-1)^{\operatorname{deg} g \operatorname{deg} m} f(m) g(n)$.

By an algebra $A$ we shall always mean a positively graded $F$-algebra with unit $\eta: F \rightarrow A$ and augmentation $\varepsilon: A \rightarrow F$. Let $I(A)=\operatorname{ker} \varepsilon$ denote the augmentation ideal.

For $L$ a left and $R$ a right $A$-module we shall need the following adjoint associativity isomorphisms:

(1.3) $\alpha:\left(R \otimes_{A} L\right)^{*} \rightarrow \operatorname{Hom}_{A}\left(R, L^{*}\right)$

given by $[\alpha(f)](r)(l)=f(r \otimes l)$.

(1.4) $\beta:\left(R \otimes_{A} L\right)^{*} \rightarrow \operatorname{Hom}_{A}\left(L, R^{*}\right)$

given by $[\beta(f)](l)(r)=(-1)^{\operatorname{deg} r \operatorname{deg} l} f(r \otimes l)$. 
If $M$ is a complex (differential module) with differential $\partial$ then $M^{*}$ has differential $\delta$ given by

(1.5) $\delta(f)(m)=(-1)^{\operatorname{deg} f+1} f \partial(m), f \in M^{*}, m \in M$.

(1.6) We now summarize some familiar facts concerning the two sided bar construction $B(A, A)$. Recall that $B(A, A)=A \otimes T(I(A)) \otimes A$, where $T$ denotes the tensor algebra. Hence $B(A, A)$ is generated by elements of the form $a \otimes a_{1} \otimes \cdots$ $\otimes a_{s} \otimes a^{\prime}$ where $a, a^{\prime} \in A$ and $a_{i} \in I(A)$. Such elements are written simply as $a\left[a_{1}|\cdots| a_{s}\right] a^{\prime}$ and are assigned bidegree $(s, t)$ where $s$ is the homological degree and $t$ is the internal degree. The total degree is $s+t$, i.e.

$$
\operatorname{deg} a\left[a_{1}|\cdots| a_{s}\right] a^{\prime}=s+\operatorname{deg} a+\sum_{i=1}^{s} \operatorname{deg} a_{i}+\operatorname{deg} a^{\prime} .
$$

Let $B_{s}(A, A)_{t}$ denote the submodule generated by elements of bidegree $(s, t)$. A differential for $B(A, A)$ is given by

$$
\begin{aligned}
\partial\left(a\left[a_{1}|\cdots| a_{s}\right] a^{\prime}\right)= & (-1)^{e_{0}} a a_{1}\left[a_{2}|\cdots| a_{s}\right] a^{\prime} \\
& +\sum_{i=1}^{s-1}(-1)^{e_{i}} a\left[a_{1}|\cdots| a_{i} a_{i+1}|\cdots| a_{s}\right] a^{\prime} \\
& -(-1)^{e_{s-1}} a\left[a_{1}|\cdots| a_{s-1}\right] a_{s} a^{\prime},
\end{aligned}
$$

where $e_{0}=\operatorname{deg} a, e_{i}=\operatorname{deg} a\left[a_{1}|\cdots| a_{i}\right]$.

Let $\bar{B}(A)=F \otimes_{A} B(A, A) \otimes_{A} F$ and for $L$ a left and $R$ a right $A$-module let $B(R, A, L)=R \otimes_{A} B(A, A) \otimes_{A} L$. Then $B(R, A, A)$ is a resolution of $R$ by free right $A$-modules and $B(A, A, L)$ is a resolution of $L$ by free left $A$-modules. Hence

$$
\begin{aligned}
\operatorname{Tor}_{*}^{A}(R, L) & =H_{*}(B(R, A, L)), \\
\operatorname{Ext}_{A}^{*}\left(R, L^{*}\right) & =H_{*}\left(\operatorname{Hom}_{A}\left(B(R, A, A), L^{*}\right)\right), \\
\operatorname{Ext}_{A}^{*}\left(L, R^{*}\right) & =H_{*}\left(\operatorname{Hom}_{A}\left(B(A, A, L), R^{*}\right)\right) .
\end{aligned}
$$

The homology and cohomology modules of $A$ are

$$
H_{*}(A)=\operatorname{Tor}_{*}^{A}(F, F), \quad H^{*}(A)=\operatorname{Ext}_{A}^{*}(F, F) .
$$

By the adjunction isomorphisms $\alpha$ and $\beta$ of (1.3), (1.4)

$$
\operatorname{Ext}_{A}^{*}\left(R, L^{*}\right)=H_{*}\left(B(R, A, L)^{*}\right), \quad \operatorname{Ext}_{A}^{*}\left(L, R^{*}\right)=H_{*}\left(B(R, A, L)^{*}\right) .
$$

Hence the cobar construction $C(R, A, L)=B(R, A, L)^{*}$ is a suitable complex for computing $\operatorname{Ext}_{A}$. We shall need an explicit expression for $C(F, A, L)=T\left(I(A)^{*}\right) \otimes L^{*}$. Let $\mu_{A}^{*}: A^{*} \rightarrow A^{*} \otimes A^{*}$ be dual to the multiplication map of $A$ and let $\mu_{L}^{*}: L^{*} \rightarrow A^{*} \otimes L^{*}$ be dual to the structure map of $L$ as a left $A$-module. If $\mu_{A}^{*}(\alpha)=\sum_{r} \alpha_{r}^{\prime} \otimes \alpha_{r}^{\prime \prime}$ and $\mu_{L}^{*}(\lambda)=\sum_{r} \alpha_{r}^{\prime} \otimes \lambda_{r}^{\prime}$ then by (1.5) the differential of $C(F, A, L)$ dual to (1.7) with $R=F$ is given by

$$
\begin{array}{r}
\delta\left(\left[\alpha_{1}|\cdots| \alpha_{n}\right] \lambda\right)=-\sum_{1 \leqq i \leqq n ; r}(-1)^{\varepsilon_{i, r}\left[\alpha_{1}|\cdots| \alpha_{i, r}^{\prime}\left|\alpha_{i, r}^{\prime \prime}\right| \cdots \mid \alpha_{n}\right] \lambda} \\
-(-1)^{\varepsilon_{n+1, r}} \sum_{r}\left[\alpha_{1}|\cdots| \alpha_{n} \mid \alpha_{r}^{\prime}\right] \lambda_{r}^{\prime}
\end{array}
$$

where $\varepsilon_{i, r}=\operatorname{deg}\left[\alpha_{1}|\cdots| \alpha_{i, r}^{\prime}\right]$ and $\varepsilon_{n+1, r}=\operatorname{deg}\left[\alpha_{1}|\cdots| \alpha_{n} \mid \alpha_{r}^{\prime}\right] \lambda_{r}^{\prime}$. 
The complex $\bar{B}(A)$ has the structure of a differential coalgebra with diagonal:

$$
D:\left[a_{1}|\cdots| a_{n}\right]=\sum_{i=0}^{n}\left[a_{1}|\cdots| a_{i}\right] \otimes\left[a_{i+1}|\cdots| a_{n}\right]
$$

The dual complex $\bar{C}(A)=\bar{B}(A) *$ is thus a differential algebra with (cup) product $\cup$ :

$$
\left[\alpha_{1}|\cdots| \alpha_{k}\right] \cup\left[\beta_{1}|\cdots| \beta_{l}\right]=\left[\alpha_{1}|\cdots| \alpha_{k}\left|\beta_{1}\right| \cdots \mid \beta_{k}\right] .
$$

Formula (1.8) shows $C(F, A, L)$ to be a differential left $\bar{C}(A)$-module.

(1.9) If $M$ is a module let $s M$ denote a bigraded copy of $M$ in which each element is given an extra degree, the homological degree, of one; i.e., if $x \in M_{n}$ then the bidegree of $s x$ is $(1, n)$.

2. Koszul algebras. In this section we define Koszul algebras, give various examples, and explicitly compute the cohomology algebras of certain Koszul algebras.

Let $T\left\{x_{i}\right\}$ denote the free associative or tensor algebra generated by the $F$-module with basis $\left\{x_{i}\right\}$ indexed over some totally ordered countable set $I$, usually thought of as the positive integers. Then $T\left\{x_{i}\right\}=\sum_{j \geqq 0} T_{j}\left\{x_{i}\right\}$ where $T_{0}\left\{x_{i}\right\}=F$ and $T_{j}\left\{x_{i}\right\}$ $=\bigotimes^{j}\left\{x_{i}\right\}$ for $j>0$; the augmentation of $T\left\{x_{i}\right\}$ is given by the natural projection $\varepsilon: T\left\{x_{i}\right\} \rightarrow T_{0}\left\{x_{i}\right\}=F$. A presentation of an algebra $A$ is an epimorphism of augmented algebras $\alpha: T\left\{x_{i}\right\} \rightarrow A$. Let $R=\operatorname{ker}(\alpha)$, then $A \cong T\left\{x_{i}\right\} \mid R$. Now $A$ is said to be a pre-Koszul algebra if it admits a presentation $\alpha$ such that $R$ is the two sided ideal generated by elements of the form $\sum_{i} f_{i} x_{i}+\sum_{j, k} f_{j, k} x_{j} x_{k}$ where $f_{i}$ and $f_{j, k}$ are in $F$. Let $\alpha\left(x_{i}\right)=a_{i}$ and observe that $a_{i} \in I(A)$. If $\left\{a_{i}\right\}$ are linearly independent then $\left\{a_{i}\right\}$ is called a pre-Koszul set of generators for $A$ and $\alpha$ is called a pre-Koszul presentation. Note that $A$ is defined by relations of the form

$$
\sum_{i} f_{i} a_{i}+\sum_{j, k} f_{j, k} a_{j} a_{k}=0 .
$$

A pre-Koszul algebra is said to be homogeneous if each $f_{i}=0$ in each relation (1). Now the tensor algebra $T\left\{x_{i}\right\}$ is filtered with $F_{p} T\left\{x_{i}\right\}$ spanned by all monomials of length $\leqq p$, with 1 assigned length 0 . If $\alpha: T\left\{x_{i}\right\} \rightarrow A$ is any presentation define $F_{p} A=\alpha\left(F_{p} T\left\{x_{i}\right\}\right)$ and $E_{p, q}^{0} A=\left(F_{p} A / F_{p-1} A\right)_{p+q}$. If $\alpha$ is a pre-Koszul presentation then $E_{p, q}^{0} A$ is called the associated homogeneous pre-Koszul algebra of $A$. Note that $E^{0} A$ is generated by images $b_{i}$ of $a_{i}$ in $E_{1, *}^{0} A$ and is defined by the relations $\sum_{j, k} f_{j, k} b_{j} b_{k}=0$. Of course $A$ is homogeneous if and only if $E^{0} A \cong A$ as algebras. In this case $A$ may be bigraded with the first degree the length.

Let $A$ be a homogeneous pre-Koszul algebra with pre-Koszul generators $\left\{a_{i}\right\}$. Since $A$ is bigraded, $H^{*} A=\operatorname{Ext}_{A}^{*}(F, F)$ is trigraded. Furthermore, the generators $\left\{a_{i}\right\}$ form a basis for the set of indecomposable elements of $A$ and so $H^{1, *, *} A$ $=H^{1,1, *} A$ has a basis $\left\{\alpha_{i}\right\}$ where $\alpha_{i} \in H^{1,1, q} A$ corresponds to $a_{i} \in A_{1, q}$. We say that $A$ is a homogeneous Koszul algebra if $H^{*} A$ is generated, as an algebra, by the set $\left\{\alpha_{i}\right\}$. This is equivalent to the condition that $H^{s, p, *} A=0$ unless $s=p$. To see this, 
observe that $\bar{C}^{p}(A)^{p, *}$ is generated by $p$-fold products of elements of $\bar{C}^{1}(A)^{1, *}$. Finally we say that a pre-Koszul algebra $A$ is a $K o s z u l$ algebra if $E^{0} A$ is a homogeneous Koszul algebra.

The following proposition is useful in recognizing Koszul algebras.

Proposition 2.1. If $A$ and $A^{\prime}$ are homogeneous Koszul algebras then $A \otimes A^{\prime}$ is also a homogeneous Koszul algebra.

Proof. Let $\left\{a_{i}\right\}$ and $\left\{a_{j}^{\prime}\right\}$ be Koszul generators for $A$ and $A^{\prime}$ respectively. Then $\left\{a_{i} \otimes 1\right\} \cup\left\{1 \otimes a_{j}^{\prime}\right\}$ generate $A \otimes A^{\prime}$ subject to

$$
\left(1 \otimes a_{j}^{\prime}\right)\left(a_{i} \otimes 1\right)=(-1)^{\operatorname{deg} a_{i} \operatorname{deg} a_{j}^{\prime}}\left(a_{i} \otimes 1\right)\left(1 \otimes a_{j}^{\prime}\right)
$$

together with the relations induced by the relations of $A$ and $A^{\prime}$. Hence $A \otimes A^{\prime}$ is a homogeneous pre-Koszul algebra. Furthermore, $H^{*}\left(A \otimes A^{\prime}\right)=H^{*} A \otimes H^{*} B$ and so $A \otimes A^{\prime}$ is a Koszul algebra.

2.2 Examples. (1) The tensor algebra $T\left\{x_{i}\right\}$ is a homogeneous pre-Koszul algebra with generators $\left\{x_{i}\right\}$ and zero relations. The elements $\left\{x_{i}\right\}$ span the indecomposables of $T\left\{x_{i}\right\}$ and (using the notation of (1.9)) $H^{*} T\left\{x_{i}\right\}=N\left\{s x_{i}\right\}$ where $N\left\{x_{i}\right\}$ is the null (or trivial) algebra generated by $\left\{x_{i}\right\}$. Hence $T\left\{x_{i}\right\}$ is a homogeneous Koszul algebra. Furthermore, $H^{*} N\left\{x_{i}\right\}=T\left\{s x_{i}\right\}$ and so $N\left\{x_{i}\right\}$ is also a homogeneous Koszul algebra.

(2) The free commutative algebra $A\left\{x_{i}\right\}$ is a homogeneous pre-Koszul algebra

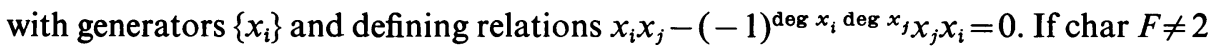
then $A\left\{x_{i}\right\}=F\left[x_{i}^{+}\right] \otimes E\left[x_{i}^{-}\right]$where $\left\{x_{i}^{+}\right\},\left\{x_{i}^{-}\right\} \subset\left\{x_{i}\right\}$ are the elements of even and odd internal degree respectively and $F\left[x^{+}\right]\left(E\left[x^{-}\right]\right)$is the polynomial (exterior) algebra. If char $F=2$ set $\left\{x_{i}^{+}\right\}=\left\{x_{i}\right\}$ and $\left\{x_{i}^{-}\right\}=\varnothing$. It is classical that

$$
H^{*} F\left[x_{i}^{+}\right]=E\left[s x_{i}^{+}\right] \text {and } H^{*} E\left[x_{i}^{-}\right]=F\left[s x_{i}^{-}\right] \text {. }
$$

Hence $F\left[x_{i}^{+}\right]$and $E\left[x_{i}^{-}\right]$are homogeneous Koszul algebras. Proposition 2.1 implies that $A\left\{x_{i}\right\}$ is also a homogeneous Koszul algebra.

(3) If $L$ is a graded Lie algebra then the universal enveloping algebra $U(L)$ is a Koszul algebra and any basis $\left\{a_{i}\right\}$ of $L$ is a Koszul set of generators. The defining re-

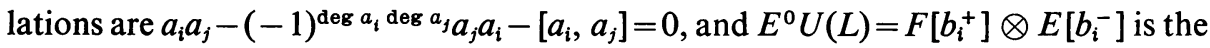
associated homogeneous Koszul algebra.

(4) If $L$ is a graded restricted Lie algebra and char $F=2$, then the universal enveloping algebra $V(L)$ is a Koszul algebra and any basis $\left\{a_{i}\right\}$ of $L$ is a Koszul set of generators. The defining relations are those of (3) and $a_{i}^{2}+\xi\left(a_{i}\right)=0$, where $\xi$ is the restriction; $E^{0} V(L)=E\left[b_{i}\right]$ is the associated homogeneous Koszul algebra.

(5) The $\bmod p$ Steenrod algebra $A$ [10] has Koszul generators $\left\{P^{i}\right\} \cup\left\{\beta P^{i}\right\}, i>0$, $j \geqq 0$ (if $p=2, P^{j}=S q^{j}$ and $\beta P^{i}, \beta$ omitted) and relations

1. $P^{0}=1$,

2. $\beta \cdot P^{i}=\beta P^{i}$,

3. $\beta^{2}=0$, and the Adem relations 
4. if $a<p b$

$$
P^{a} \cdot P^{b}=\sum_{t=0}^{[a / p]}(-1)^{a+t}\left(\begin{array}{c}
(p-1)(b-t)-1 \\
a-p t
\end{array}\right) P^{a+b-t} \cdot P^{t},
$$

5. if $a \leqq p b$ then

$$
\begin{aligned}
P^{a} \cdot \beta P^{b}= & \sum_{t=0}^{[a / p]}(-1)^{a+t}\left(\begin{array}{c}
(p-1)(b-t) \\
a-p t
\end{array}\right) \beta P^{a+b-t} \cdot P^{t} \\
& +\sum_{t=0}^{[(a-1) / p]}(-1)^{a+t-1}\left(\begin{array}{c}
(p-1)(b-t)-1 \\
a-p t-1
\end{array}\right) P^{a+b-t} \cdot \beta P^{t},
\end{aligned}
$$

where the binomial coefficients are taken $\bmod p$ and $[x]$ is the largest integer $\leqq x$. Since $P^{0}=1, A$ is not homogeneous. The next example shows that $A$ is actually a Koszul algebra.

(6) The $\bmod p$ Steenrod algebra $A_{L}$ for simplicial restricted Lie algebras (see $[4,7.1,8.8]$ for the case $p=2$ ) is a homogeneous Koszul algebra (the proof of this is given in 5.3). In fact $A_{L}$ has the same generators and relations as (5) except $P^{0}=0$. Therefore $A_{L}$ is isomorphic to the associated $E^{0} A$ of (5) and hence $A$ is a Koszul algebra.

(7) Let $A$ be the $Z_{2}$-algebra generated by 4 letters $\{a, b, c, d\}$ with defining relations $a c=c d=0$ and $b c=c b$. Then $[a b|c| d] \in \bar{B}(A)$ is a nonbounding 3-cycle of length 4. Hence $A$ is an example of a homogeneous pre-Koszul algebra which is not Koszul.

2.3. We shall now show that the cohomology algebra of a homogeneous Koszul algebra admits a very explicit description.

Suppose $\left\{a_{i}\right\}_{i \in I}$ is a Koszul set of generators for an algebra $A$. Let $B$ be an $F$-module basis for $A$ consisting of $1, a_{i}$, and certain monomials $a_{i_{1}} \cdots a_{i_{n}}, a_{i,} \in\left\{a_{i}\right\}$. A set

$$
S \subset U=\bigcup_{n=1}^{\infty} I_{1} \times \cdots \times I_{n}, \quad I_{i}=I,
$$

is called a labeling set for $B$ if for each $a \in B-\{1\}$ there is a unique $\left(i_{1}, \ldots, i_{n}\right) \in S$ such that $a=a_{i_{1}} \cdots a_{i_{n}}$. The pair $(B, S)$ is called a labeled basis for $A$. Clearly such bases exist. Suppose further that $A$ is a homogeneous Koszul algebra. Then each monomial $a_{k} a_{l}$ of $A$ has a unique expression of the form

$$
a_{k} a_{l}=\sum_{(i, j) \in S} f\left(\begin{array}{l}
k, l \\
i, j
\end{array}\right) a_{i} a_{j}
$$

where the summation is taken over all $i, j$ such that $(i, j) \in S$. We shall call (2.4) the admissible relations for $A$ (with respect to $(B, S))$. Let $B^{*}=\left\{1, \alpha(i), \alpha\left(i_{1}, \ldots, i_{n}\right)\right\}$ denote the dual basis, i.e., if $\left(i_{1}, \ldots, i_{n}\right) \in S-\{1\}$ and $a \in A$ then

$$
\begin{aligned}
\left\langle\alpha\left(i_{1}, \ldots, i_{n}\right), a\right\rangle & =1 & & \text { if } a=a_{i_{1}} \cdots a_{i_{n}}, \\
& =0 & & \text { otherwise. }
\end{aligned}
$$


In keeping with our previous notation $\alpha_{i}$ will denote the cohomology class of the cocycle $[\alpha(i)] \in \bar{C}^{1}(A)^{1, *}$.

TheOREM 2.5. Let $A$ be a homogeneous Koszul algebra with Koszul generators $\left\{a_{i}\right\}_{i \in I}$, labeled basis $(B, S)$, and admissible relations (2.4). Then the cohomology algebra $H^{*} A$ is generated by $\left\{\alpha_{i}\right\}_{i \in I}$ subject to: if $(i, j) \in S$ then

$$
(-1)^{v_{i, j} \alpha_{i} \alpha_{j}}+\sum_{(k, l) \in U-S}(-1)^{v_{k, l} f}\left(\begin{array}{l}
k, l \\
i, j
\end{array}\right) \alpha_{k} \alpha_{l}=0
$$

where $\nu_{u, v}=\operatorname{deg} \alpha_{u}+\left(\operatorname{deg} \alpha_{u}-1\right)\left(\operatorname{deg} \alpha_{v}-1\right)$.

Proof. By hypothesis $H^{*} A$ is generated (as an algebra) by $\left\{\alpha_{i}\right\}$. To determine the relations among these classes observe that $\bar{C}^{p-1}(A)^{p}$ is spanned by elements of the form $\left[\alpha\left(i_{1}\right)|\cdots| \alpha\left(i_{j}, i_{j+1}\right)|\cdots| \alpha\left(i_{p}\right)\right]$ where exactly one factor has length 2 and the remaining factors are cycles of length 1 . Thus the relations of $H^{*}(A)$ are defined by $\delta[\alpha(i, j)]=0$ for $\alpha(i, j) \in B^{*}$. Applying (2.4) and (1.8) we have

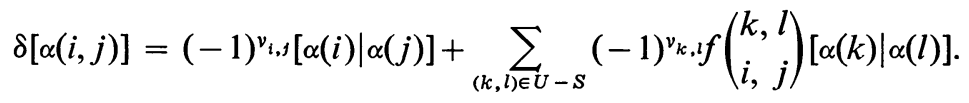

The signs are introduced by the map $\theta$ of (1.2).

3. Koszul complexes and resolutions. Let $A$ be a Koszul algebra with a fixed Koszul set of generators $\left\{a_{i}\right\}, i \in I$. Let $R$ and $L$ be right and left $A$-modules respectively. The main purpose of this section is to define the Koszul complex $K_{*}(R, A, L)$, a small subcomplex of the bar construction $B_{*}(R, A, L)$, whose homology is $\operatorname{Tor}_{*}^{A}(R, L)$. This is done in (3.7). In case $R=F=L, K_{*}(F, A, F)$ is shown to be a differential subcoalgebra of $B_{*}(F, A, F)=\bar{B}_{*}(A)$.

The splitting $1-\eta \circ \varepsilon: A \hookrightarrow I(A)$ induces a natural injection $i: E_{1}^{0} A \hookrightarrow I(A)$ which extends to a natural injection

$$
i: \bar{B}_{p}\left(E^{0} A\right)_{p} \hookrightarrow \bar{B}_{p}(A) .
$$

Each element of $H_{p, p}\left(E^{0} A\right)$ may be represented by a cycle of $\bar{B}_{p}\left(E^{0} A\right)_{p}$ of the form $\sum_{i} f_{i}\left[b_{i_{1}}|\cdots| b_{i_{p}}\right]$ where $b_{i_{j}} \in E_{1}^{0} A$ and $i\left(b_{i_{j}}\right) \in\left\{a_{i}\right\}$. This representation is unique because there exist no nonzero $(p+1)$-chains of length $p(a(p+1)$-chain has length at least $p+1)$. Hence there is a natural injection

$$
j: H_{p, p}\left(E^{0} A\right) \hookrightarrow B_{p}\left(E^{0} A\right)_{p} .
$$

Finally the composition $i \circ j$ induces a natural injection of modules

$$
\text { ı: } R \otimes H_{p, p}\left(E^{0} A\right) \otimes L \hookrightarrow B_{p}(R, A, L)
$$

given explicitly by

$$
\iota\left(r \otimes\left\{\sum_{i} f_{i}\left[b_{i_{1}}|\cdots| b_{i_{p}}\right]\right\} \otimes l\right)=r \otimes \sum_{i} f_{i}\left[a_{i_{1}}|\cdots| a_{i_{p}}\right] \otimes l .
$$

We shall presently define the Koszul complex to be the module $R \otimes H_{*, *}\left(E^{0} A\right) \otimes L$ 
with a differential such that (3.3) is a map of complexes. First we need to analyze the nonbounding cycles of $\bar{B}\left(E^{0} A\right)$ more carefully. For each integer $k>0$, let

$$
I^{k}=I_{1} \times \cdots \times I_{k}, \quad I_{i}=I,
$$

where $I$ is the indexing set for $\left\{a_{i}\right\}$.

LEMMA 3.2. For $b=\sum_{i} f_{i}\left[b_{i_{1}}|\cdots| b_{i_{p}}\right]$ to be a cycle of $\bar{B}_{p}\left(E^{0} A\right)_{p}$ it is necessary and sufficient that, for each $j(1 \leqq j \leqq p-1)$ and each $\left(k_{1}, \ldots, k_{j-1}\right) \in I^{j-1},\left(k_{j+2}, \ldots, k_{p}\right)$ $\in I^{p-j-1}$, the following condition holds

$$
\sum_{i}(-1)^{e_{i j}} f_{i} b_{i,} b_{i_{j+1}}=0
$$

where $e_{i_{1}}=\operatorname{deg}\left[b_{i_{1}}|\cdots| b_{i_{1}}\right]$ and where the summation is taken over all $i$ such that $\left(i_{1}, \ldots, i_{j-1}\right)=\left(k_{1}, \ldots, k_{j-1}\right)$ and $\left(i_{j+1}, \ldots, i_{p}\right)=\left(k_{j+1}, \ldots, k_{p}\right)$.

Proof. Since $E^{0} A$ is bigraded, $\bar{B}\left(E^{0} A\right)=\bar{B}_{s}\left(E^{0} A\right)_{p, q}$ is trigraded (see 1.6). Hence

$$
\partial b=\sum_{i} \sum_{j=1}^{p-1}(-1)^{e_{i f}} f_{i}\left[b_{i_{1}}|\cdots| b_{i_{j}} b_{i_{j}+1}|\cdots| b_{i_{p}}\right]
$$

where

$$
\begin{aligned}
& {\left[b_{i_{1}}|\cdots| b_{i}, b_{i_{j+1}}|\cdots| b_{i_{p}}\right] \in \bar{B}_{p-1}\left(E^{0} A\right)_{p}} \\
& \quad=E_{1}^{0} A_{1} \otimes \cdots \otimes E_{1}^{0} A_{j-1} \otimes E_{2}^{0} A \otimes E_{1}^{0} A_{1} \otimes \cdots \otimes E_{1}^{0} A_{p-j-1},
\end{aligned}
$$

(each $\left.A_{i}=A\right)$. Hence $\partial b=0$ if and only if for each $j(1 \leqq j \leqq p-1)$ and each $\left(k_{1}, \ldots, k_{j-1}\right) \in I^{j-1}$, we have

$$
\sum_{i}(-1)^{e_{i j}} f_{i}\left[b_{i_{1}}|\cdots| b_{i_{j}} b_{i_{j+1}}|\cdots| b_{i_{p}}\right]=0
$$

where the summation is taken over all $i$ such that $\left(i_{1}, \ldots, i_{j-1}\right)=\left(k_{1}, \ldots, k_{j-1}\right)$ and $\left(i_{j+2}, \ldots, i_{p}\right)=\left(k_{j+2}, \ldots, k_{p}\right)$. But this is equivalent to

$$
\sum_{i}(-1)^{e_{i j}} f_{i} b_{i j} b_{i_{j+1}}=0
$$

summed over the same values of $i$.

COROLlaRY 3.3. If $c=\sum_{i} f_{i}\left[b_{i_{1}}|\cdots| b_{i_{p}}\right]$ is a cycle of $\bar{B}_{p}\left(E^{0} A\right)_{p}, k=\left(k_{1}, \ldots, k_{m}\right) \in I^{m}$, and $k^{\prime}=\left(k_{m+1}, \ldots, k_{p}\right) \in I^{p-m}$ then

$$
c_{k}=\sum_{i ;\left(i_{1}, \ldots, i_{m}\right)=k} f_{i}\left[b_{i_{m+1}}|\cdots| b_{i_{p}}\right]
$$

is a cycle of $\bar{B}_{p-m}\left(E^{0} A\right)_{p-m}$ and

$$
c_{k^{\prime}}=\sum_{i ;\left(i_{m}+1, \ldots, i_{p}\right)=k^{\prime}} f_{i}\left[b_{i_{1}}|\cdots| b_{i_{m}}\right]
$$

is a cycle of $\bar{B}_{m}\left(E^{0} A\right)_{m}$. 
Proof. Let $e_{i}=\operatorname{deg}\left[b_{i_{1}}|\cdots| b_{i_{j}}\right]$. Then

$$
\begin{aligned}
& \partial c_{k^{\prime}}=\sum_{j=1}^{m-1} \sum_{\left(i_{m+1}, \ldots, i_{p}\right)=k^{\prime}}(-1)^{e_{i,}} f_{i}\left[b_{i_{1}}|\cdots| b_{i_{i}} b_{i_{j}+1}|\cdots| b_{i_{m}}\right] \\
& =\sum_{j=1}^{m-1} \sum_{\begin{array}{c}
\left(l_{1}, \ldots, l_{-1}\right) \in I^{j-1} \\
\left(l_{j+2}, \ldots, l_{m}\right) \in I^{m-j-1}
\end{array}}\left[b_{l_{i}}|\cdots| b_{l_{j-1}}\left|\sum_{i}(-1)^{e_{i j}} f_{i} b_{i_{j}} b_{i_{j+1}}\right| b_{l_{j+2}}|\cdots| b_{l_{m}}\right]
\end{aligned}
$$

where the innermost summation is taken over all $i$ such that $\left(i_{1}, \ldots, i_{j-1}\right)=$ $\left(l_{1}, \ldots, l_{j-1}\right),\left(i_{j+2}, \ldots, i_{m}, i_{m+1}, \ldots, i_{p}\right)=\left(l_{j+2}, \ldots, l_{m}, k_{m+1}, \ldots, k_{p}\right)$. Since $\partial c=0$, Lemma 3.2 implies that each of the summations over $i$ is zero. Hence $\partial c_{k^{\prime}}=0$. A similar argument shows $\partial c_{k}=0$. This completes the proof.

Suppose $x \in H_{p, p}\left(E^{0} A\right)$ is represented by $\sum_{i} f_{i}\left[b_{i_{1}}|\cdots| b_{i_{p}}\right]$. Since

$$
\partial\left(\sum_{i} f_{i}\left[b_{i_{1}}|\cdots| b_{i_{p}}\right]\right)=0
$$

in $\bar{B}\left(E^{0} A\right)$ it follows that in $\bar{B}(A)$

$$
\begin{aligned}
\partial\left(\sum_{i} f_{i}\left[a_{i_{1}}|\cdots| a_{i_{p}}\right]\right) & =\sum_{i} \sum_{j=1}^{p-1}(-1)^{e_{i j}} f_{i}\left[a_{i_{1}}|\cdots| a_{i_{j}} a_{i_{j+1}}|\cdots| a_{i_{p}}\right] \\
& =\sum_{i} \sum_{j=1}^{p-1} \sum_{k} f_{i_{j k}}\left[a_{i_{1}}|\cdots| a_{i_{j k}}|\cdots| a_{i_{p}}\right]
\end{aligned}
$$

for certain $f_{i_{j k}} \in F$ and $a_{i, k} \in\left\{a_{i}\right\}$. Now define a differential $d$ for $R \otimes H_{*, *}\left(E^{0} A\right) \otimes L$ by

$$
\begin{aligned}
d(r \otimes x \otimes l)= & \sum_{m}(-1)^{\operatorname{deg} r} r a_{m} \otimes\left\{\sum_{i, i_{1}=m}\left[b_{i_{2}}|\cdots| b_{i_{p}}\right]\right\} \otimes l \\
& +(-1)^{\operatorname{deg} r} r \otimes\left\{\sum_{j=1}^{p-1} \sum_{i, k} f_{i_{j k}}\left[b_{i_{1}}|\cdots| b_{i_{j k}}|\cdots| b_{i_{p}}\right]\right\} \otimes l \\
& -\sum_{m}(-1)^{\operatorname{deg} r+e_{p-1} r} \otimes\left\{\sum_{i, i_{p}=m}\left[b_{i_{1}}|\cdots| b_{i_{p-1}}\right]\right\} \otimes a_{m} l .
\end{aligned}
$$

It follows from Lemma 3.1 and Corollary 3.3 that each of the bracketed expressions in this formula is a cycle of $B\left(E^{0} A\right)$. It is clear from (3.1), (3.4), (3.5) and (1.7) that $\partial \circ i=i \circ d$. Hence $d^{2}=0$.

3.6. ExAmple. To illustrate (3.5) let $A$ be the mod 2 Steenrod algebra (see (2.2) Example 5) and let $R=Z_{2}=L$. Then the cycle

$$
x=\left\{\left[S q^{2}\left|S q^{2}\right| S q^{3}\right]+\left[S q^{2}\left|S q^{4}\right| S q^{1}\right]+\left[S q^{5}\left|S q^{1}\right| S q^{1}\right]\right\}
$$

of $\bar{B}_{3}\left(E^{0} A\right)_{3,4}$ represents an element of $H_{3,3,4}\left(E^{0} A\right)$. Using the Adem relations $S q^{2} S q^{2}=0, S q^{2} S q^{3}=S q^{5}+S q^{4} S q^{1}, S q^{2} S q^{4}=S q^{6}+S q^{5} S q^{1}$, formula (3.5) yields $d x=\left\{\left[S q^{2} \mid S q^{5}\right]+\left[S q^{6} \mid S q^{1}\right]\right\}$, a cycle of $\bar{B}_{2}\left(E^{0} A\right)_{2,5}$.

3.7. The Koszul complex $K_{*}(R, A, L)$ is the complex with module $K_{p}(R, A, L)$ $=R \otimes H_{p, p}\left(E^{0} A\right) \otimes L$ and with differential $d_{p}$ given by (3.5). The following is the main theorem of our theory. 
THEOREM 3.8. If $A$ is a Koszul algebra then $\operatorname{Tor}_{*, *}^{A}(R, L)$ is the homology of the Koszul complex $K_{*}(R, A, L)$.

The proof is given in $\$ 10$.

Since $\operatorname{Tor}^{A}(A, L)=L$ the complex $K_{*}(A, A, L)$ is a resolution of $L$ by free left $A$-modules. We call $K_{*}(A, A ; L)$ the Koszul resolution of $L$. Similarly $K_{*}(R, A, A)$ is the Koszul resolution of $R$ (by free right $A$-modules).

Using (3.7) we may restate (3.1) as

Proposition 3.9. There is a canonical injection of complexes

given by

$$
\text { ı: } K_{*}(R, A, L) \hookrightarrow B_{*}(R, A, L)
$$

$$
\iota\left(r \otimes\left\{\sum_{i} f_{i}\left[b_{i_{1}}|\cdots| b_{i_{p}}\right]\right\} \otimes l\right)=r \otimes \sum_{i} f_{i}\left[a_{i_{1}}|\cdots| a_{i_{p}}\right] \otimes l .
$$

Following the notational convention $\bar{B}_{*}(A)=B_{*}(F, A, F)$ let $\bar{K}_{*}(A)$ denote $K_{*}(F, A, F)$. The complex $\bar{K}_{*}(A)$ inherits the structure of a differential coalgebra from $\bar{B}_{*}(A)$ via the following commutative diagram

$$
\begin{aligned}
& H_{p, p}\left(E^{0} A\right)=Z\left(\bar{B}_{p}\left(E^{0} A\right)_{p}\right) \stackrel{j}{\longrightarrow} B_{p}\left(E^{0} A\right)_{p} \longrightarrow \quad i \quad \bar{B}_{p}(A) \\
& Z\left(\sum_{r+s=p} \bar{B}_{r}\left(E^{0} A\right)_{r} \otimes \bar{B}_{s}\left(E^{0} A\right)_{s}\right) \hookrightarrow \sum_{r+s=p} \bar{B}_{r}\left(E^{0} A\right)_{r} \otimes \bar{B}_{s}\left(E^{0} A\right)_{s} \stackrel{i \otimes i}{\longrightarrow} \sum_{r+s=p} \bar{B}_{r}(A) \otimes \bar{B}_{s}(A) \\
& \approx \uparrow h \quad j \otimes j / \\
& \sum_{r+s=p} H_{r, r}(A) \otimes H_{s, s}(A)
\end{aligned}
$$

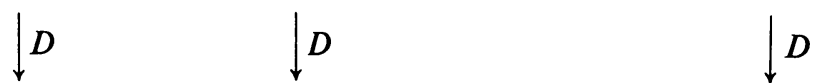

where $Z(\cdot)$ denotes the submodule of cycles and $h$ denotes the external homology product which is an isomorphism by the Künneth theorem. Let $\Delta=h^{-1} \circ D$, then

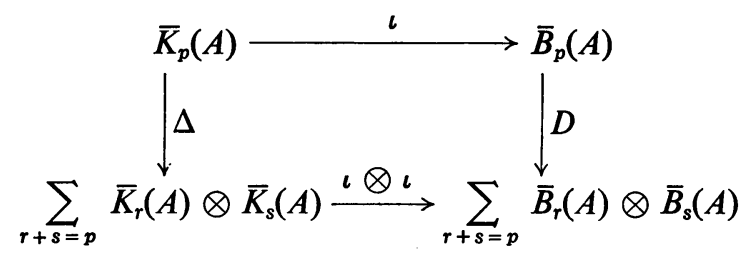

commutes and since $\iota$ is an injection it follows that $\bar{K}_{*}(A)$ is a differential coalgebra. Hence we may state

Proposition 3.10. The map $\iota: \bar{K}_{*}(A) \rightarrow \bar{B}_{*}(A)$ of Proposition 3.9 is an injection of differential coalgebras.

4. Co-Koszul complexes. In this section we obtain complexes for computing cohomology algebras and modules for Koszul algebras. Formally, these complexes 
are dual to those studied in $\S 3$; however, they seem to be more tractable. Again let $A$ be a Koszul algebra with a fixed Koszul set of generators $\left\{a_{i}\right\}, i \in I$, and let $R$ and $L$ be right and left $A$-modules respectively.

The co-Koszul complex $K^{*}(R, A, L)$ is defined to be the graded $F$-dual of $K_{*}(R, A, L)$. Since $A$ is assumed to be locally finite over a field $F, K^{*}(R, A, L)$ is a complex for computing $\operatorname{Ext}_{A}^{*}\left(L, R^{*}\right)=\operatorname{Tor}_{*}^{A}(R, L)^{*}$. We shall first describe the general structure of this complex and delay an explicit description until 4.4.

In case $R=F=L$ let $\bar{K}^{*}(A)$ denote $K^{*}(F, A, F)=H^{*}\left(E^{0} A\right)$, the graded $F$-dual of $\bar{K}_{*}(A)=K_{*}(F, A, F)=H_{*}\left(E^{0} A\right)$. Now $\bar{C}\left(E^{0} A\right)$ is generated as an algebra by the elements of $\bar{C}^{1}\left(E^{0} A\right)^{1}=\left(E_{1}^{0} A\right)^{*}$-each of which is a cycle-and so it follows that there is a natural projection $\rho: \bar{C}^{p}\left(E^{0} A\right)^{p} \rightarrow \bar{C}^{p}\left(E^{0} A\right)^{p} / \operatorname{Im}^{p}=H^{p, p}\left(E^{0} A\right)$. Furthermore, the isomorphism $\theta$ of (1.2) induces an isomorphism $\theta^{\prime}: H^{p, p}\left(E^{0} A\right) \rightarrow$ $H_{p, p}\left(E^{0} A\right)^{*}$ such that the following diagram of algebra morphisms commutes

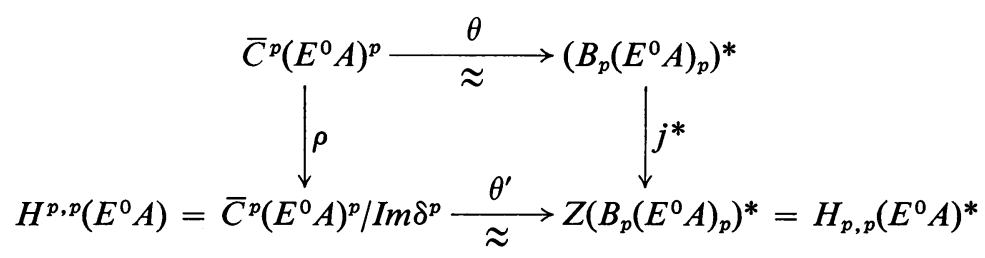

where $j^{*}$ is dual to $j$ of (3.2). Hence the algebra structure of $\bar{K}^{*}(A)$ inherited from $H^{*, *}\left(E^{0} A\right)$ is dual to the coalgebra structure of $\bar{K}_{*}(A)$. Thus the differential $\delta$ of $\bar{K}^{*}(A)$ is determined by its action on $\bar{K}^{1}(A)=\bar{C}^{1}\left(E^{0} A\right)^{1}$. Let $\bar{\mu}_{A}$ be the composition

$$
\left(E_{1}^{0} A\right)^{*} \stackrel{\pi^{*}}{\longrightarrow} I(A)^{*} \stackrel{\mu_{A}^{*}}{\longrightarrow} I(A)^{*} \otimes I(A)^{*} \stackrel{i^{*} \otimes i^{*}}{\longrightarrow}\left(E_{1}^{0} A\right)^{*} \otimes\left(E_{1}^{0} A\right)^{*}
$$

where $\pi: I(A) \rightarrow E_{1}^{0} A$ is an $F$-splitting of $i$ obtained by choosing an $F$-basis for $A$ which extends $\left\{a_{i}\right\}$ and where $\mu_{A}$ is the multiplication map of $A$. Then using (3.5), (1.2) and (1.5) we find that for $\beta \in \bar{K}^{1}(A)$

$$
\delta(\beta)=\sum_{j}(-1)^{\operatorname{deg} \beta_{j}^{\prime} \beta_{j}^{\prime} \cdot \beta_{j}^{\prime \prime}}
$$

where $\bar{\mu}_{A}(\beta)=\sum_{j} \beta_{j}^{\prime} \otimes \beta_{j}^{\prime \prime}$.

We shall now obtain the structure of $K^{*}(F, A, L)$, leaving to the reader the obvious generalization to $K^{*}(R, A, L)$. Let $\bar{\mu}_{L}$ be the composition

$$
L^{*} \stackrel{\mu_{L}^{*}}{\longrightarrow} A^{*} \otimes L^{*} \stackrel{i^{*} \otimes L^{*}}{\longrightarrow}\left(E_{1}^{0} A\right)^{*} \otimes L^{*}
$$

where $\mu_{L}$ is the structure map of $L$ as a left $A$-module. Choose an $F$-basis for $L$ and thus a dual basis for $L^{*}$. Now for $\lambda \in L^{*}$, let

$$
\Delta_{L}(\lambda)=\sum_{j}(-1)^{\operatorname{deg} \beta_{j} \beta_{j}} \otimes \lambda_{j}
$$

where $\mu_{L}^{*}(\lambda)=\sum_{j} \beta_{j} \otimes \lambda_{j}$. Dualizing (3.5) for $K_{*}(F, A, L)$ we see that $K^{*}(F, A, L)$ $=\bar{K}^{*}(A) \otimes L^{*}$ is a differential left $\bar{K}^{*}(A)$-module and $\delta(1 \otimes \lambda)=\Delta_{L}(\lambda)$. Summarizing 
TheOREM 4.3. Let $A$ be a Koszul algebra then

(1) The cohomology algebra $H^{*}(A)$ is the homology of the co-Koszul complex $\bar{K}^{*}(A)$. As a differential algebra, $\bar{K}^{*}(A)=H^{*}\left(E^{0} A\right)$ with differential $\delta$ determined by (4.1). If $L$ is a left A-module then

(2) The module $\operatorname{Ext}_{A}^{*}(L, F)$ is the homology of the co-Koszul complex $K^{*}(F, A, L)$. As a differential left $\bar{K}^{*}(A)$-module, $K^{*}(F, A, L)=\bar{K}^{*}(A) \otimes L^{*}$ with differential $\delta$ determined by (4.2).

Proof. Part (1) is implied by Theorems 3.8 and 3.9 and duality. Part (2) follows from the preceding paragraph.

4.4. Applying Theorem 2.5 we see that the algebra structure of $\bar{K}^{*}(A)=H^{*}\left(E^{0} A\right)$ admits a very explicit description; using (4.1) we can extend this description to include the differential algebra structure of $\bar{K}^{*}(A)$. Let $\left(B_{A}, S\right)$ be a labeled basis for $A$ (see (2.3)). We can assume the relations for $A$ are written in admissible form with respect to $\left(B_{A}, S\right)$, i.e.,

$$
a_{k} a_{l}=\sum_{m} f\left(\begin{array}{c}
k, l \\
m
\end{array}\right) a_{m}+\sum_{(i, j) \in S} f\left(\begin{array}{l}
k, l \\
i, j
\end{array}\right) a_{i} a_{j}
$$

where $f\left(\begin{array}{c}k, l \\ m\end{array}\right)$ and $f\left(\begin{array}{c}k, l \\ i, j\end{array}\right)$ are in $F$. Let $B_{A}^{*}$ denote the dual basis for $A^{*}$. A basis $B_{E^{0}}{ }_{A}$ for $E^{0} A$ is formally obtained from $B_{A}$ by replacing the letter $a$ with the letter $b$ and similarly $B_{E}^{*_{0}{ }_{A}}$ from $B_{A}^{*}$ by replacing $\alpha$ with $\beta$. Admissible relations for $E^{0} A$ with respect to $\left(B_{E^{0}{ }_{A}}, S\right)$ are

$$
b_{k} b_{l}=\sum_{(i, j) \in S} f\left(\begin{array}{l}
k, l \\
i, j
\end{array}\right) b_{i} b_{j}
$$

Let $\beta_{i}$ denote the cohomology class of $[\beta(i)] \in \bar{C}^{1}\left(E^{0} A\right)^{1, *}$.

THEOREM 4.6. Let $A$ be a Koszul algebra with Koszul generators $\left\{a_{i}\right\}, i \in I$, labeled basis $\left(B_{A}, S\right)$, and admissible relations (4.5). Then the co-Koszul complex $\bar{K}^{*}(A)$ is the differential algebra generated by $\left\{\beta_{i}\right\}, i \in I$, with

(1) a relation for each $(i, j) \in S$

where

$$
(-1)^{v_{i, s} \beta_{i} \beta_{j}}+\sum_{(k, l) \in U-S}(-1)^{v_{k, l} f}\left(\begin{array}{l}
k, l \\
i, j
\end{array}\right) \beta_{k} \beta_{l}=0
$$

(2) differential

$$
\nu_{u, v}=\operatorname{deg} \beta_{u}+\left(\operatorname{deg} \beta_{u}-1\right)\left(\operatorname{deg} \beta_{v}-1\right) \quad \text { and } \quad U=\bigcup_{n=1}^{\infty} I^{n}
$$

$$
\delta \beta_{m}=\sum_{(k, l) \in U-S}(-1)^{v_{k, l} l f}\left(\begin{array}{c}
k, l \\
m
\end{array}\right) \beta_{k} \beta_{l} .
$$

Proof. Since $E^{0} A$ is by definition a homogeneous Koszul algebra, the algebra structure (1) of $\bar{K}^{*}(A)=H^{*}\left(E^{0} A\right)$ is given by Theorem 2.5. To evaluate the differential apply (4.1) noting that

$$
\bar{\mu}_{A}\left(\beta_{m}\right)=\sum_{m}(-1)^{\left(\operatorname{deg} \beta_{k}-1\right)\left(\operatorname{deg} \beta_{l}-1\right)} f\left(\begin{array}{c}
k, l \\
m
\end{array}\right) \beta_{k} \otimes \beta_{l} .
$$

The signs are introduced by $\theta$ of (1.2). 
5. A sufficient condition. In order to apply the preceding theory one must have effective means to determine whether a homogeneous pre-Koszul algebra is in fact Koszul. The following sufficient condition is often easy to use since it is completely algebraic and requires no homological calculations.

Let $A$ be a homogeneous pre-Koszul algebra with pre-Koszul generators $\left\{a_{i}\right\}$, $i \in I$. Let $(B, S)$ be a labeled basis for $A$ (see 2.3); if $a \in B,\left(i_{1}, \ldots, i_{n}\right) \in S$ and $a=a_{i_{1}} \cdots a_{i_{n}}$ then $\left(i_{1}, \ldots, i_{n}\right)$ is called the $\left(S\right.$-) label of $a$. The set $U=\bigcup_{n=1}^{\infty} I^{n}$ is ordered first by length and then by the lexicographical ordering derived from the order of $I$. Since $S \subset U, S$ inherits an ordering.

5.1. We call $(B, S)$ a Poincaré-Birkhoff-Witt (PBW) basis if

(1) $\left(i_{1}, \ldots, i_{k}\right)$ and $\left(j_{1}, \ldots, j_{l}\right) \in S$ implies that $\left(i_{1}, \ldots, i_{k}, j_{1}, \ldots, j_{l}\right) \in S$ or else the label of each monomial appearing in the admissible expression (see 2.4) of $a=a_{i_{1}} \cdots a_{i_{k}} a_{j_{1}} \cdots a_{j_{k}}$ is strictly greater than $\left(i_{1}, \ldots, i_{k}, j_{1}, \ldots, j_{l}\right)$,

(2) for $k>2,\left(i_{1}, \ldots, i_{k}\right) \in S$ if and only if for each $j(1 \leqq j<k)\left(i_{1}, \ldots, i_{j}\right) \in S$ and $\left(i_{j+1}, \ldots, i_{k}\right) \in S$.

If $A^{*}$ has a PBW basis then $A$ is called a Poincaré-Birkhoff-Witt (PBW) algebra.

5.2. ExAMPLES. The following algebras of (2.2) are PBW algebras

(1) $T\left\{x_{i}\right\}$ with $B=\left\{1\right.$, all monomials $\left.x_{i_{1}} \otimes \cdots \otimes x_{i_{n}}\right\}$,

(2) $F\left[x_{i}\right]$ with $B=\left\{1\right.$, monomials $\left.x_{n}^{e_{n}} x_{n-1}^{e_{n}} \cdots x_{1}^{e_{1}} ; e_{i}=0,1,2, \ldots\right\}$,

(3) $E\left[x_{i}\right]$ with $B=\left\{1\right.$, monomials $\left.x_{n}^{\varepsilon_{n}} x_{n-1}^{\varepsilon_{n}-1} \cdots x_{1}^{\varepsilon_{1}} ; \varepsilon_{i}=0,1\right\}$,

$$
\begin{array}{r}
A_{L} \text { with } B=\left\{1 \text {, monomials }\left(\beta^{\varepsilon_{0}} P^{s_{1}}\right)\left(\beta^{\varepsilon_{1}} P^{s_{2}}\right) \cdots\left(\beta^{\varepsilon_{k-1}} P^{s_{k}}\right) \beta^{\varepsilon_{k}}\right. \\
\left.s_{i}=1,2, \ldots, \varepsilon_{i}=0,1, s_{i} \geqq p s_{i+1}+\varepsilon_{i}\right\} \\
\text { (if } \left.p=2 \operatorname{set} \varepsilon_{i}=0\right) .
\end{array}
$$

TheOREM 5.3. If $A$ is a PBW algebra then $A$ is a homogeneous Koszul algebra.

The proof of 5.3, which is a generalization of that given in $[8,8.4]$ for $A_{L}$, is given below. The idea is to filter the cobar construction $C^{s, p}=\bar{C}^{s}(A)^{p, *}$ using the labels of a fixed PBW basis $(B, S)$ for $A$ and to show that off the diagonals $s=p$ the quotients of this filtration have trivial homology. It then follows from a standard argument that $H^{s, p}(A)=0$ unless $s=p$. First, however, we indicate several changes in $[8,8.4]$ necessary to account for signs and the fact that we are no longer using the special basis of $A_{L}$. Let $B^{*}=\left\{1, \alpha(i), \alpha\left(i_{1}, \ldots, i_{n}\right)\right\}$ denote the basis for $A^{*}$ dual to $B$ (see 2.3 ).

If $I$ is a label of length $p$ then define $F_{I} C^{s, p}=$ submodule generated by the elements

$$
\left[\alpha\left(i_{1}, \ldots, i_{k_{1}}\right)\left|\alpha\left(i_{k_{1}+1}, \ldots, i_{k_{2}}\right)\right| \cdots \mid \alpha\left(i_{k_{s-1}+1}, \ldots, i_{p}\right)\right]
$$

where $\alpha\left(i_{1}, \ldots, i_{k_{1}}\right), \ldots, \alpha\left(i_{k_{s-1}+1}, \ldots, i_{p}\right) \in B^{*}$ and $I \geqq\left(i_{1}, \ldots, i_{p}\right)$. This defines a complete increasing filtration of $C^{* * *}$ as a module. For each label $I$ it is easy to see (using condition (1) of 5.1) that $F_{I} C^{* * *}$ is a subcomplex. Let $F_{I-1} C=\bigcup_{J<I} F_{J} C$. Then $\delta$ induces a map

$$
\delta: F_{I} C / F_{I-1} C \rightarrow F_{I} C / F_{I-1} C
$$


which by 1.8 and (1) and (2) of 5.1 is given by (let $k_{0}=0, k_{s}=p$ )

$$
\begin{aligned}
& \delta\left[\alpha\left(i_{1}, \ldots, i_{k_{1}}\right)|\cdots| \alpha\left(i_{k_{s-1}+1}, \ldots, i_{p}\right)\right]
\end{aligned}
$$

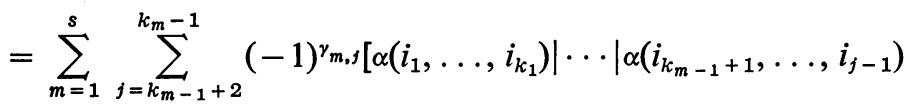

$$
\begin{aligned}
& \left.\left|\alpha\left(i_{j}, \ldots, i_{k_{m}}\right)\right| \cdots \mid \alpha\left(i_{k_{s-1}+1}, \ldots, i_{p}\right)\right]\left(\bmod F_{I-1} C\right)
\end{aligned}
$$

where

$$
\begin{aligned}
\gamma_{m, j}= & \operatorname{deg}\left[\alpha\left(i_{1}, \ldots, i_{k_{1}}\right)|\cdots| \alpha\left(i_{k_{m-1}+1}, \ldots, i_{j-1}\right)\right] \\
& +\operatorname{deg} \alpha\left(i_{k_{m-1}}, \ldots, i_{j-1}\right) \cdot \operatorname{deg} \alpha\left(i_{j}, \ldots, i_{k_{m}}\right) .
\end{aligned}
$$

5.4. Proof of Theorem 5.2. Fix $p>0$ and let $1 \leqq s \leqq p$. To each element

$$
x=\left[\alpha\left(i_{1}, \ldots, i_{k_{1}}\right)\left|\alpha\left(i_{k_{1}+1}, \ldots, i_{k_{2}}\right)\right| \cdots \mid \alpha\left(i_{k_{s-1}+1}, \ldots, i_{p}\right)\right]
$$

of length $p$ and homological degree $s$ we assign an integer ai $(x)$, called the admissibility index, defined as follows: Let ai $(x)$ be the smallest integer $k_{j}, 1 \leqq j \leqq s-1$, such that $\alpha\left(i_{k_{j-1}+1}, \ldots, i_{k_{j}+1}, \ldots, i_{k_{j+1}}\right) \in B^{*}$. If no such integer exists then set ai $(x)=p$. Now define a contracting homotopy

$$
\Phi: F_{I} C^{s, p} / F_{I-1} C^{s, p} \rightarrow F_{I} C^{s-1, p} / F_{I-1} C^{s-1, p}
$$

for $1 \leqq s \leqq p$ by the following formula:

If

$$
x=\left[\alpha\left(i_{1}\right)\left|\alpha\left(i_{2}\right)\right| \cdots\left|\alpha\left(i_{j}\right)\right| \alpha\left(i_{j+1}, \ldots, i_{k_{j+1}}\right)|\cdots| \alpha\left(i_{k_{s-1}+1}, \ldots, i_{p}\right)\right]
$$

with ai $(x)=j$ and $k_{j}=j<p$ set

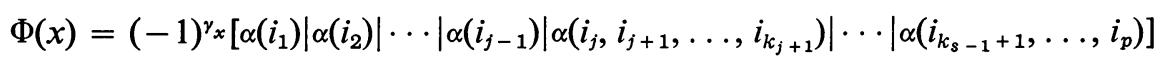

where

$$
\gamma_{x}=\operatorname{deg}\left[\alpha\left(i_{1}\right)|\cdots| \alpha\left(i_{j}\right)\right]+\operatorname{deg} \alpha\left(k_{j}\right) \operatorname{deg} \alpha\left(i_{j+1}, \ldots, i_{k_{j+1}}\right) .
$$

Otherwise set $\Phi(x)=0$.

A straightforward verification now shows that if $0<s<p$ and $x \in F_{I} C^{s, p} / F_{I-1} C^{s, p}$ then $\delta \Phi(x)+\Phi \delta(x)=x$.

6. Applications to universal enveloping algebras. In this section we derive the Koszul resolution $K_{*}(F, U(L), U(L))$ for a universal enveloping algebra $U(L)$. The resolution coincides with May's generalization of the classical Koszul resolution. Comparisons are also given for $L$, a restricted Lie algebra over a field of characteristic $p=2$.

6.1. Graded Lie algebras. Let $L$ be a graded Lie algebra [6] (including, of course, the classical case of $L$ concentrated in degree zero). In 2.2, Example (3), the universal enveloping algebra $U(L)$ is defined and shown to be a Koszul algebra with associated homogeneous Koszul algebra $E^{0} U(L)=E\left(L^{-}\right) \otimes A\left(L^{+}\right)$where $E\left(L^{-}\right)$is the exterior algebra on the elements $L^{-} \subset L$ of odd order and $A\left(L^{+}\right)$is the free 
commutative algebra on the elements $L^{+} \subset L$ of even order. Recall that a typical element $\left\langle x_{1}, \ldots, x_{n}\right\rangle \in E\left(L^{-}\right)$has bidegree $\left(n, \sum_{i=1}^{n} \operatorname{deg} x_{i}-n\right)$ and similarly a typical element $x_{1} \cdots x_{n} \in A\left(L^{+}\right)$has bidegree $\left(n, \sum_{i=1}^{n} \operatorname{deg} x_{i}-n\right)$.

Using the notation (1.9), $E\left(s L^{+}\right)$is a trigraded $F$-algebra with a typical element $\left\langle s x_{1}, \ldots, s x_{n}\right\rangle \in E\left(s L^{+}\right)$having tridegree $\left(n, n, \sum_{i=1}^{n} \operatorname{deg} x_{i}-n\right)$. Similarly the divided power algebra $\Gamma\left(s L^{-}\right)$is a trigraded $F$-algebra with a typical element $\gamma_{r_{1}}\left(s x_{1}\right) \cdots \gamma_{r_{n}}\left(s x_{n}\right) \in \Gamma\left(s L^{-}\right)$having tridegree $(p, p, q)$ where $p=\sum_{i=1}^{n} r_{i}$ and $q=\sum_{i=1}^{n} r_{i} \operatorname{deg} x_{i}-p$. We may consider $\Gamma\left(s L^{-}\right)$and $E\left(s L^{+}\right)$as commutative differential $F$-algebras with zero differential and with the homological degree given by the first gradation. The complexes $\bar{B}\left(E\left(L^{-}\right)\right)$and $\bar{B}\left(A\left(L^{+}\right)\right)$are also commutative differential $F$-algebras under the shuffle product [3, Theorems 12.1-12.2] and it is classical that the differential algebra maps

$$
g_{1}: \Gamma\left(s L^{-}\right) \rightarrow \bar{B}\left(E\left(L^{-}\right)\right)
$$

defined by $g_{1}\left(\gamma_{r}(s x)\right)=[x|\cdots| x]$ (r factors) and

$$
g_{2}: E\left(s L^{+}\right) \rightarrow \bar{B}\left(A\left(L^{+}\right)\right)
$$

defined by $g_{2}\langle s x\rangle=[x]$ induce homology isomorphisms

$$
g_{1^{*}}: \Gamma\left(s L^{-}\right) \stackrel{\approx}{\longrightarrow} H_{*}\left(E\left(L^{-}\right)\right), \quad g_{2^{*}}: E\left(s L^{+}\right) \stackrel{\approx}{\longrightarrow} H_{*}\left(A\left(L^{+}\right)\right) .
$$

Now the shuffle product also induces a map [3, p. 313]

given by

$$
g_{3}: \bar{B}\left(E\left(L^{-}\right)\right) \otimes \bar{B}\left(A\left(L^{+}\right)\right) \stackrel{\approx}{\longrightarrow} \bar{B}\left(E\left(L^{-}\right) \otimes A\left(L^{+}\right)\right)
$$

$$
g_{3}\left(\left[x_{1}|\cdots| x_{n}\right] \otimes\left[y_{1}|\cdots| y_{m}\right]\right)=(-1)^{m n}\left[x_{1}|\cdots| x_{n}\right] *\left[y_{1}|\cdots| y_{m}\right]
$$

which yields a homology isomorphism

$$
g_{3^{*}}: H_{*}\left(E\left(L^{-}\right)\right) \otimes H_{*}\left(A\left(L^{+}\right)\right) \stackrel{\approx}{\longrightarrow} H_{*}\left(E\left(L^{-}\right) \otimes A\left(L^{+}\right)\right) .
$$

By the definition 3.7

$$
K_{*}(F, U(L), U(L))=H_{*}\left(E\left(L^{-}\right) \otimes A\left(L^{+}\right)\right) \otimes U(L) .
$$

Hence we have the following isomorphism of complexes

$$
g_{*}: \Gamma\left(s L^{-}\right) \otimes E\left(s L^{+}\right) \otimes U(L) \stackrel{\approx}{\longrightarrow} K_{*}(F, U(L), U(L))
$$

where $g_{*}=\left(g_{3^{*}} \otimes U(L)\right) \circ\left(g_{1^{*}} \otimes g_{2^{*}} \otimes U(L)\right)$ and where the differential of the left-hand side is dictated by the differential of $K_{*}(F, U(L), U(L))$. Now (6.2) and the embedding of Proposition 3.9 result in an embedding

$$
\Gamma\left(s L^{-}\right) \otimes E\left(s L^{+}\right) \otimes U(L) \rightarrow B(F, U(L), U(L))
$$

which coincides with that described by May [4, p. 143]. Hence (6.2) is an isomorphism between May's $Y(L)=\Gamma\left(s L^{-}\right) \otimes E\left(s L^{+}\right) \otimes U(L)$ and the Koszul resolution $K_{*}(F, U(L), U(L))$. 
6.3. Graded restricted Lie algebras (char $F=2$ ). Let $L$ be a graded restricted Lie algebra [6] over a field of characteristic $p=2$. In 2.2, Example (4), the universal enveloping algebra $V(L)$ is defined and shown to be a Koszul algebra with associated homogeneous Koszul algebra $E^{0} V(L)=E(L)$. The Koszul resolution is defined (3.7) and

$$
K_{*}(F, V(L), V(L))=H_{*}(E(L)) \otimes V(L)=\Gamma(s L) \otimes V(L) .
$$

The embedding of Proposition 3.9 again shows that $K_{*}(F, V(L), V(L))$ agrees with May's resolution $X(L)$ [4, Remark 10, p. 140].

7. Application to the Steenrod algebra. Let $A$ denote the $\bmod p$ Steenrod algebra (2.2, Example (5)). In this section we compute the co-Koszul complexes $\bar{K}^{*}(A)$ for $\operatorname{Ext}_{A}\left(Z_{p}, Z_{p}\right)$ and $K^{*}\left(Z_{p}, A, H^{*} X\right)$ for $\operatorname{Ext}_{A}\left(H^{*} X, Z_{p}\right)$ where $X$ is a simplicial spectrum [1] with finitely generated reduced cohomology groups $H^{*} X$ with coefficients in $Z_{p}$. These complexes provide a homological description of the results of [1].

7.1. The prime 2. Let $p=2$, then with respect to the generators $\left\{S q^{1}, S q^{2}, S q^{3}, \ldots\right\}$ $A$ is a Koszul algebra. This is immediate because the associated graded algebra $E^{0} A$ (2.2, Example (6)) has a PBW basis $B=\left\{S q^{a_{k}} \ldots S q^{a_{1}}: a_{i+1} \geqq 2 a_{i}, k \geqq 1\right\}$ (5.2, Example (4)) and hence is a homogeneous Koszul algebra by Theorem 5.3.

The mod 2 Adem (defining) relations for $A$ are

$$
S q^{a} S q^{b}=\left(\begin{array}{c}
b-1 \\
a
\end{array}\right) S q^{a+b}+\sum_{j=1}^{[a / 2]}\left(\begin{array}{c}
b-j-1 \\
a-2 j
\end{array}\right) S q^{a+b-j} S q^{j}
$$

for $0 \leqq a<2 b$. If we also let $S q^{i}$ denote the image of $S q^{i}$ in $E^{0} A$ then the relations of $E^{0} A$ are

$$
S q^{a} S q^{b}=\sum_{j=1}^{[a / 2]}\left(\begin{array}{c}
b-j-1 \\
a-2 j
\end{array}\right) S q^{a+b-j} S q^{j} .
$$

Let $B^{*}$ be the basis dual to $B$ and let $\sigma_{i}$ denote the element $\left[S q^{i *}\right] \in H^{1,1, i-1}\left(E^{0} A\right)$ where $S q^{i *} \in B^{*}$ is dual to $S q^{i}$. Now Theorem 4.6 shows that as a differential $Z_{2}$-algebra $\bar{K}^{*}(A)$ is generated by $\sigma_{i}, i>0$, subject to the relations: if $a \geqq 2 b>0$

$$
\sigma_{a} \cdot \sigma_{b}=\sum_{j=2 b}^{\{2(a+b) / 3\}}\left(\begin{array}{c}
a-j-1 \\
j-2 b
\end{array}\right) \sigma_{j} \cdot \sigma_{a+b-j}
$$

with differential

$$
\delta \sigma_{a}=\sum_{j=1}^{\{2 a / 3\}}\left(\begin{array}{c}
a-j-1 \\
j
\end{array}\right) \sigma_{j} \cdot \sigma_{a-j}
$$

where $\{x\}$ is the greatest integer less than $x$.

It is now easy to see that there is an anti-isomorphism of differential $Z_{2}$-algebras $\Phi:\left(\bar{K}^{*}(A), \delta\right) \rightarrow\left(E^{1} S, d^{1} S\right)$ extending the map of generators given by $\sigma_{i+1} \mapsto \lambda_{i}$ $(i \geqq 0)$, where $\left(E^{1} S, d^{1} S\right)$, known as the $\Lambda$ algebra, is the $\left(E^{1}, d^{1}\right)$ term of the 
Adams spectral sequence (derived from the lower 2-central series filtration) for the simplicial group sphere spectrum FS [1]. One merely checks that the relations and differential given above carry over to those described by Wang [13, 1.5.4, 1.6.5]

$$
\begin{aligned}
\lambda_{i} \lambda_{2 i+1+n} & =\sum_{j \geqq 0}\left(\begin{array}{c}
n-j-1 \\
j
\end{array}\right) \lambda_{i+n-j} \lambda_{2 i+1+j}, \\
d^{1} \lambda_{n} & =\sum_{j \geqq 0}\left(\begin{array}{c}
n-j-1 \\
j+1
\end{array}\right) \lambda_{n-j-1} \lambda_{j} .
\end{aligned}
$$

More generally if $L$ is a left $A$-module (of finite type over $Z_{2}$ ) then by Theorem 4.3, $K^{*}\left(Z_{2}, A, L\right)$ is the differential left $\bar{K}^{*}(A)$-module $\bar{K}^{*}(A) \otimes L^{*}$ with differential determined by

$$
\delta(1 \otimes \lambda)=\Delta_{L}(\lambda), \quad \lambda \in L^{*},
$$

where $\Delta_{L}$ is induced by the coproduct of $L^{*}$ as a left $A^{*}$-comodule (see 4.2). Now $L^{*}$ has the structure of a right $A$-module (transposed to the left $A$-module structure of $L$ ), using this structure

$$
\Delta_{L}(\lambda)=\sum_{i>0} \sigma_{i} \otimes \lambda S q^{i}, \quad \lambda \in L^{*} .
$$

In particular if $L=H^{*} X$ for a simplicial spectrum $X$ with finitely generated cohomology groups we have an anti-isomorphism $\Psi$ (extending $\Phi$ ) of differential left $\bar{K}^{*}(A)$-modules and differential right $E^{1} S=\Phi\left(\bar{K}^{*}(A)\right)$-modules

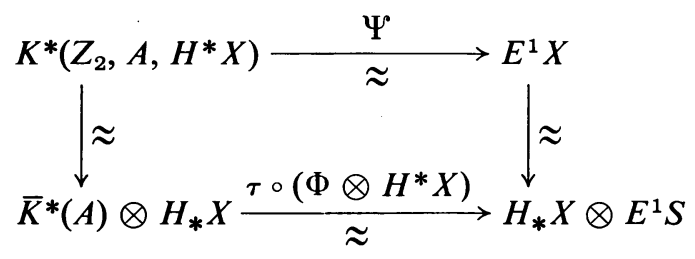

where $\tau: B \otimes B^{\prime} \rightarrow B^{\prime} \otimes B$ is the twisting map for $Z_{2}$-modules.

.Setting $X=A S$, the simplicial Eilenberg-MacLane $K\left(Z_{2}\right)$ spectrum, we see that the co-Koszul resolution $K^{*}\left(Z_{2}, A, A\right)$ corresponds to $\left(E^{1} A S, d^{1} A S\right)$.

The relationship between $\bar{K}^{*}(A)$ and the cobar complex $\bar{C}^{*}(A)$ is given by

$$
\iota^{*}: \bar{C}^{*}(A) \rightarrow \bar{K}^{*}(A),
$$

the surjective map of differential algebras which is dual to the injection $\iota: \bar{K}_{*}(A)$ $\rightarrow \bar{B}_{*}(A)$ of Proposition 3.10. In order to explicitly describe $\imath^{*}$, recall that $\bar{C}^{*}(A)=T\left(s I\left(A^{*}\right)\right)$ with diagonal differential $\delta^{*}$ and that according to Milnor $A^{*}=Z_{2}\left[\xi_{1}, \xi_{2}, \ldots\right]$ where $\xi_{i}$ is dual to $S q^{2^{2-1}} S q^{2^{i-2}} \cdots S q^{1}$ with respect to the basis

$B$. On the generators of $\bar{C}^{*}(A), \iota^{*}$ is given by

$$
\begin{aligned}
\iota^{*}([x]) & =\sigma_{n} & & \text { if } x=\xi_{1}^{n}, n>0, \\
& =0 & & \text { otherwise. }
\end{aligned}
$$

Thus $\imath^{*}\left[\xi_{1}^{a}|\cdots| \xi_{1}^{a}\right]=\sigma_{a_{1}} \cdots \sigma_{a_{n}}$. 
A simple calculation shows that for $m>0, n \geqq 0$,

$$
\begin{aligned}
\iota^{*} \delta^{*}\left[\xi_{2}^{m} \xi_{1}^{n}\right] & =\iota^{*}\left[\left(\xi_{1}^{2 m} \otimes \xi_{1}^{m}\right) \cdot\left(1 \otimes \xi_{1}+\xi_{1} \otimes 1\right)^{n}\right] \\
& =\sum_{i=0}^{n}\left(\begin{array}{l}
n \\
i
\end{array}\right) \sigma_{2 m+i} \sigma_{m+n-i}=0
\end{aligned}
$$

which under the anti-isomorphism $\Phi$ yields the original presentation of the defining relations $[1, \S 2.4($ iii) $]$

$$
\sum_{i+j=n}\left(\begin{array}{c}
i+j \\
i
\end{array}\right) \lambda_{i-1+m} \lambda_{j-1+2 m}=0 .
$$

Setting $m=0$, one obtains the differential $d^{1} S[1, \S 2.4(\mathrm{iv})]$

$$
d^{1} \lambda_{n-1}=\sum_{i+j=n}\left(\begin{array}{c}
i+j \\
i
\end{array}\right) \lambda_{i-1} \lambda_{j-1} \quad(n \geqq 2) .
$$

Hence the elements of $\bar{C}^{*}(A)$ involving $\xi_{i}, i>2$, are not necessary for determining the cohomology algebra $H^{*}(A)$ and the co-Koszul complex $\bar{K}^{*}(A)$ is obtained by factoring out (in the precise manner stated above) these superfluous elements.

7.2. The odd primes. Let $p$ be an odd prime. Then $A$ is a Koszul algebra with generators $\left\{P^{i}\right\} \cup\left\{\beta P^{j}\right\}, i>0, j \geqq 0(2.2$, Examples (5), (6)). A PBW-basis $B$ for $A$ is described in (5.2, Example (4)). Let $B^{*}$ be the basis dual to $B$ and let $\pi_{i}$ denote the element $\left[P^{i *}\right] \in H^{1,1,2 i(p-1)-1}\left(E^{0} A\right)$ where $P^{i *} \in B^{*}$ is dual to $P^{i}$. Similarly let $\rho_{j}$ denote the element $\left[\beta P^{j *}\right] \in H^{1,1,2 j(p-1)}\left(E^{0} A\right)$ where $\beta P^{j *} \in B^{*}$ is dual to $\beta P^{j}$.

The co-Koszul complex $\bar{K}^{*}(A)$ is most easily described by analyzing the surjective map of differential algebras $\imath^{*}: \bar{C}^{*}(A) \rightarrow \bar{K}^{*}(A)$ transposed to the inclusion of Proposition 3.10. Since $A^{*}=Z_{p}\left[\xi_{1}, \xi_{2}, \ldots\right] \otimes E\left[\tau_{0}, \tau_{1}, \ldots\right]$, $\iota^{*}$ is given on the generators of $\bar{C}^{*}(A)$ by

$$
\begin{array}{rlrl}
\iota^{*}([x])=\pi_{i} & \text { if } x=\xi_{1}^{i}, i>0, \\
& =\rho_{j} & & \text { if } x=\tau_{0} \xi_{1}^{j}, j \geqq 0, \\
& =0 & & \text { otherwise. }
\end{array}
$$

The classes $\pi_{i}, \rho_{j}$ generate $\bar{K}^{*}(A)$ and the relations are now obtained by evaluating $\iota^{*} \delta^{*}$ on the classes $\left[\tau_{0} \xi_{2}^{m} \xi_{1}^{n}\right],\left[\xi_{2}^{m} \xi_{1}^{n}\right], m \geqq 1, n \geqq 0$, and $\left[\tau_{0} \tau_{1} \xi_{2}^{m} \xi_{1}^{n}\right],\left[\tau_{1} \xi_{2}^{m} \xi_{1}^{n}\right], m \geqq 0$, $n \geqq 0$. (Here $\delta^{*}$ is the differential of $\bar{C}^{*}(A)$.) For example,

hence

$$
\iota^{*} \delta^{*}\left(\left[\tau_{0} \xi_{2}^{m} \xi_{1}^{n}\right]\right)=\sum_{i+j=n}\left(\begin{array}{c}
i+j \\
i
\end{array}\right) \iota *\left(\left[-\xi^{p m+j} \mid \tau_{0} \xi_{1}^{m+i}\right]+\left[\xi_{1}^{p m+j} \tau_{0} \mid \xi_{1}^{m+i}\right]\right)=0
$$

$$
\sum_{i+j=n}\left(\begin{array}{c}
i+j \\
i
\end{array}\right)\left(\pi_{p m+j} \cdot \rho_{m+i}-\rho_{p m+j} \cdot \pi_{m+i}\right)=0 \quad(m \geqq 1, n \geqq 0) .
$$

The other relations are obtained similarly

$$
\sum_{i+j=n}\left(\begin{array}{c}
i+j \\
i
\end{array}\right) \pi_{p m+j} \cdot \pi_{m+n-i}=0 \quad(m \geqq 1, n \geqq 0),
$$




$$
\begin{aligned}
\sum_{i+j=n}\left(\begin{array}{c}
i+j \\
i
\end{array}\right) \pi_{p m+j+1} \rho_{m+i} & =0 \quad(m, n \geqq 0), \\
\sum_{i+j=n}\left(\begin{array}{c}
i+j \\
i
\end{array}\right) \rho_{p m+j+1} \cdot \rho_{m+i} & =0 \quad(m, n \geqq 0) .
\end{aligned}
$$

The differential of $\bar{K}^{*}(A)$ is obtained by evaluating $i^{*} \delta^{*}$ on $\xi_{1}^{n}$ and $\tau_{0} \xi_{1}^{n}$

$$
\begin{aligned}
& \delta \pi_{n}=\sum_{i+j=n}\left(\begin{array}{c}
i+j \\
i
\end{array}\right) \pi_{j} \pi_{i} \quad(n>0), \\
& \delta \rho_{n}=\sum_{i+j=n}\left(\begin{array}{c}
i+j \\
i
\end{array}\right)\left(\pi_{j} \rho_{i}-\rho_{j} \pi_{i}\right) \quad(n \geqq 0) .
\end{aligned}
$$

Results (1)-(6) correspond to those of $\left[1, \S 2.4^{\prime}\right]$ under the anti-isomorphism of differential $Z_{p}$-algebras $\Phi:\left(\bar{K}^{*}(A), \delta\right) \rightarrow\left(E^{1} S, d^{1} S\right)$ extending the map on generators given by $\pi_{i+1} \mapsto \lambda_{i}, \rho_{i} \mapsto-\mu_{i-1}(i \geqq 0)$.

8. Matric Massey products. We now briefly examine the role of matric Massey products in the preceding theory. Recall [12] that for an algebra $A$ there is a 1-1 correspondence between any basis for $H_{1} A$ and a minimal set of generators for $A$ and between any basis for $\mathrm{H}_{2} \mathrm{~A}$ and a minimal set of defining relations for $A$. Hence it is clear that if $A$ is a homogeneous pre-Koszul algebra then $H^{1, p} A=0$ for $p>1$ and $H^{2, p} A=0$ for $p>2$. Generally, however, matric Massey products may appear (see 2.2, Example (7)) and a homogeneous pre-Koszul algebra need not be a homogeneous Koszul algebra. A basic result of May's theory of matric Massey products [5] states that every indecomposable element of $H^{*} A$ can be built up via matric Massey products from the indecomposable elements of $A$. In this description matric Massey products take values in the indecomposable elements of $H^{*} A$ and so there is no indeterminacy. A trivial consequence of May's result is

Proposition 8.1. Let $A$ be a homogeneous pre-Koszul algebra. In order for $A$ to be a homogeneous Koszul algebra it is necessary and sufficient that every matric Massey product of $H^{*} A$ be zero.

9. Duality. For certain homogeneous Koszul algebras $A$ the algebra $H^{*} H^{*} A$ may be naturally identified with $A$. In this section we examine this duality phenomenon and its relationship to the dual Adams spectral sequence for the sphere spectrum for simplicial Lie algebras.

For any bigraded algebra $A$ let $D^{s, q} A=H^{s, s, q} A$. We call $D^{*}$ the diagonal cohomology functor; clearly $D^{*} A$ is a subalgebra of $H^{*} A$. Note also that $D^{*} A$ is a homogeneous pre-Koszul algebra.

Proposition 9.1. There is a natural map of bigraded algebras $\Phi: D^{*} D^{*} A \rightarrow A_{*}$ extending the natural isomorphism $\varphi: A_{1, q}^{* *} \rightarrow A_{1, q}$. If $A$ is a homogeneous pre-Koszul algebra then $\Phi$ is an isomorphism. 
9.2. ExAmples. It follows from 2.2, Example (1), that $D^{*} D^{*} T\left\{x_{i}\right\}=T\left\{x_{i}\right\}$ and $D^{*} D^{*} N\left\{x_{i}\right\}=N\left\{x_{i}\right\}$. Similarly in 2.2 , Example (2), $D^{*} D^{*} F\left[x_{i}^{+}\right]=F\left[x_{i}^{+}\right]$and $D^{*} D^{*} E\left[x_{i}^{+}\right]=E\left[x_{i}^{+}\right]$.

If $A$ is a homogeneous pre-Koszul algebra then $A$ is Koszul provided $H^{*}$ reduces to $D^{*}$ in the obvious sense. Thus with this abuse of notation:

Corollary 9.3. If $A$ and $H^{*} A$ are homogeneous Koszul algebras then

$$
\Phi: H^{*} H^{*} A \stackrel{\approx}{\longrightarrow} A .
$$

Proof of Proposition 9.1. Since $D^{*} A$ is a homogeneous pre-Koszul algebra, it suffices to show that the natural isomorphism $\varphi: A_{1, q}^{* *} \rightarrow A_{1, q}$ extends to a map of algebras $\Phi_{s}: D^{s, q} D^{*} A \rightarrow A_{s, q}$ for $s=1$ and 2 .

In degree 1 let

$$
\Phi_{1}=\varphi: D^{1, q} D^{*} A=A_{1, q}^{* *} \stackrel{\approx}{\longrightarrow} A_{1, q} .
$$

Now consider the exact sequence

$$
0 \longrightarrow R \stackrel{i}{\longrightarrow} A_{1, q} \otimes A_{1, q} \stackrel{\mu}{\longrightarrow} A_{2,2 q}
$$

where $\mu$ is the multiplication map and $R$ is the set of relations for $A_{2, *}$. The dual exact sequence shows that $R^{*}=D^{2, *} A$ and $i^{*}$ is the Yoneda multiplication map $D^{1, *} A \otimes D^{1, *} A \rightarrow D^{2, *} A$.

Thus the commutative diagram

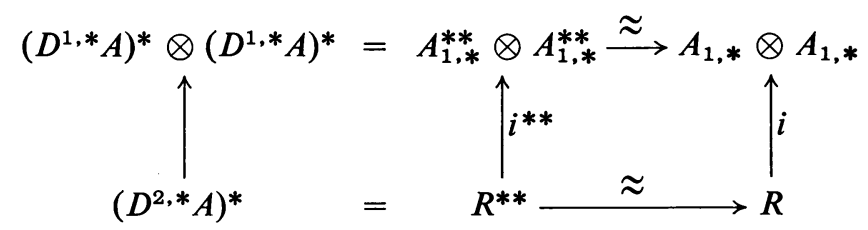

shows that $\varphi \otimes \varphi$ induces a natural injective map $\Phi_{2}: D^{2, q} D^{*} A \rightarrow A_{2, q}$ and hence the desired natural map of algebras $\Phi_{*}: D^{*} D^{*} A \rightarrow A_{*}$.

If $A$ is a homogeneous pre-Koszul algebra then $\Phi$ is surjective since $A$ is generated by elements of $A_{1, *}$. Furthermore $\Phi$ is injective since the relations of $A$ are generated by the relations of $A_{2, *}$.

9.4. ExAMPLE. Let $A_{L}$ be the mod 2 Steenrod algebra for simplicial Lie algebras (see 2.2, Example (6)). A PBW basis for $H^{*} A_{L}=\operatorname{Ext}_{A_{L}}\left(Z_{2}, Z_{2}\right)$ is given in [8, 8.3]; hence by Corollary $9.3 H^{*} H^{*} A_{L} \underset{\rightarrow}{\rightarrow} A_{L}$.

By $[8,9.1], \operatorname{Ext}_{A_{L}}\left(Z_{2}, Z_{2}\right) \stackrel{\approx}{\rightarrow} \pi_{*} L A S$, the stable stems for simplicial Lie algebras, thus

$$
\operatorname{Ext}_{\pi \cdot L A S}\left(Z_{2}, Z_{2}\right) \stackrel{\approx}{\longrightarrow} A_{L} .
$$

Geometrically, the analogous situation for the stable stems $\pi_{*} S$ has been noted by Moss [7, p. 192]: There is a dual Adams spectral sequence

$$
E_{2}=\operatorname{Ext}_{\pi_{*} S}\left(Z_{2}, Z_{2}\right) \Rightarrow A
$$

in which $E_{2} \neq E_{\infty}$ where $A$ is the $\bmod 2$ Steenrod algebra. 
10. Proof of Theorem 3.8. The proof depends on a spectral sequence: For $L$ a left and $R$ a right $A$-module, the filtration of $A$ induces a filtration of the bar construction $B(R, A, L)$ which results in a spectral sequence,

$$
E_{*, *}^{1}(R, A, L)=R \otimes H_{*, *}\left(E^{0} A\right) \otimes L \Rightarrow \operatorname{Tor}_{*, *}^{A}(R, L),
$$

the $\left(E^{1}, d^{1}\right)$ term of which is shown to coincide with the Koszul complex $K_{*}(R, A, L)$. Since $E^{0} A$ is a homogeneous Koszul algebra $\left(H_{s, p}\left(E^{0} A\right)=0\right.$ for $\left.s \neq p\right)$ the spectral sequence collapses $\left(E^{2}=E^{\infty}\right)$ thus completing the proof.

The filtration of $A$ is defined in terms of a fixed presentation $\alpha$ (see $\S 2$ ). Using this filtration define an increasing filtration of the bar construction by

$$
\begin{aligned}
& F_{p} B_{r}(R, A, L)=0 \text { for } p<0, \\
& F_{p} B_{0}(R, A, L)=R \otimes L \text { for } p \geqq 0, \\
& F_{p} B_{r}(R, A, L)=\sum_{i_{1}+\cdots+i_{r} \leqq p} R \otimes F_{i_{1}} I(A) \otimes \cdots \otimes F_{i_{r}} I(A) \otimes L \quad \text { for } p \geqq 0, r>0 .
\end{aligned}
$$

Clearly $F_{p} B(R, A, L) \subset B(R, A, L)$ is a subcomplex and $\left\{F_{p} B(R, A, L)\right\}$ is complete and bounded below. Denote by $\left\{E^{r}(R, A, L), d^{r}\right\}$ the spectral sequence associated with this filtration. The $E^{0}$ term is

$$
E_{s, p, q}^{0}(R, A, L)=\left(\frac{F_{p} B_{s}(R, A, L)}{F_{p-1} B_{s}(R, A, L)}\right)_{p+q}
$$

where $s$ is the homological degree, $p$ is the filtration degree and $p+q$ is the degree associated with the internal degrees of $R, A$, and $L$. As an $F$-module

$$
E_{s, p, q}^{0}(R, A, L)=\sum_{i+j+k=q} R_{i} \otimes \bar{B}_{s}\left(E^{0} A\right)_{p, j} \otimes L_{k} .
$$

Examining the boundary $\partial_{B(R, A, L)}$ of $B(R, A, L)$ of $(1.7)$, it is easy to see that the differential $d^{0}$ is given on a class $r \otimes x \otimes l \in R \otimes \bar{B}\left(E^{0} A\right) \otimes L$ by

$$
d^{0}(r \otimes x \otimes l)=(-1)^{\mathrm{deg} r} r \otimes \partial_{\bar{B}\left(E_{A}^{0}\right)}(x) \otimes l .
$$

Hence

$$
E_{s, p, q}^{1}(R, A, L)=\sum_{i+j+k=q} R_{i} \otimes H_{s, p, j}\left(E^{0} A\right) \otimes L_{k}
$$

and so as $F$-modules $E_{*}^{1}(R, A, L)=K_{*}(R, A, L)$. Now $d^{1}$ is the connecting homomorphism in homology of the short exact sequences

$$
0 \rightarrow \frac{F_{p-1} B(R, A, L)}{F_{p-2} B(R, A, L)} \rightarrow \frac{F_{p} B(R, A, L)}{F_{p-2} B(R, A, L)} \rightarrow \frac{F_{p} B(R, A, L)}{F_{p-1} B(R, A, L)} \rightarrow 0 .
$$

Using (3.3) and (1.7), $d^{1}$ is seen to have the form of (3.5); hence $E_{*}^{1}(R, A, L)$ $=K_{*}(R, A, L)$ as complexes.

To show that $E^{2}=E^{\infty}$ observe that

$$
d_{s, p, q}^{r}: E_{s, p, q}^{r}(R, A, L) \rightarrow E_{s-1, p-r, q+r}^{r}(R, A, L)
$$


and that

$$
E_{s, p, q}^{1}(R, A, L)=\sum_{i+j+k=q} R_{i} \otimes H_{s, p, j}\left(E^{0} A\right) \otimes L_{k}=0
$$

unless $s=p$ and so $d^{r}=0$ for $r \geqq 2$.

\section{REFERENCES}

1. A. K. Bousfield, E. B. Curtis, D. M. Kan, D. G. Quillen, D. L. Rector and J. W. Schlesinger, The mod-p lower central series and the Adams spectral sequence, Topology 5 (1966), 331-342. MR 33 \#8002.

2. H. Cartan and S. Eilenberg, Homological algebra, Princeton Univ. Press, Princeton, N. J., 1956. MR 17, 1040.

3. S. MacLane, Homology, Die Grundlehren der math. Wissenschaften, Band 114, Academic Press, New York, and Springer-Verlag, Berlin, 1963. MR 28 \#122.

4. J. P. May, The cohomology of restricted Lie algebras and of Hopf algebras, J. Algebra 3 (1966), 123-146. MR 33 \#1347.

5. - The algebraic Eilenberg-Moore spectral sequence, (to appear).

6. J. Milnor and J. C. Moore, On the structure of Hopf algebras, Ann. of Math. (2) 81 (1965), 211-264. MR 30 \#4259.

7. R. M. F. Moss, On the composition pairing of Adams spectral sequences, Proc. London Math. Soc. (3) 18 (1968), 179-192. MR 36 \#3360.

8. S. B. Priddy, Primary cohomology operators for simplicial Lie algebras, Illinois J. Math. 14 (1970).

9. —- Koszul resolutions and the Steenrod algebra, Bull. Amer. Math. Soc. 76(1970), 834839.

10. N. E. Steenrod and D. B. A. Epstein, Cohomology operations, Ann. of Math. Studies, no. 50, Princeton Univ. Press, Princeton, N. J., 1962. MR 26 \#3056.

11. J. Tate, Homology of noetherian rings and local rings, Illinois J. Math. 1 (1957), 14-27. MR 19, 119.

12. C. T. C. Wall, Generators and relations for the Steenrod algebra, Ann. of Math. (2) 72 (1960), 429-444. MR 22 \#7117.

13. John S. P. Wang, On the cohomology of the mod-2 Steenrod algebra and the non-existence of elements of Hopf invariant one, Illinois J. Math. 11 (1967), 480-490. MR 35 \#4917.

NORTHWESTERN UNIVERSITY,

EVANSTON, ILLINOIS 60201 